\title{
Therapie der \\ Demenzerkrankungen - ein Update
}

Georg Adler

Zentralinstitut für Seelische Gesundheit, Mannheim

psychoneuro 2005; 31 (10): 490-494

An der Alzheimer-Demenz sind auch vaskuläre Faktoren beteiligt. Die zerebralen Mikrogefäße scheinen eine wichtige Rolle bei der Entstehung der Erkrankung zu spielen. Bei der Diagnostik sollten daher auch eine eventuell vorliegende Hypertension, Schilddrüsenparameter, Diabetes mellitus und andere Risikofaktoren überprüft werden. Bei der Behandlung ist ein multimodaler Behandlungsansatz empfehlenswert. Zur medikamentösen Therapie stehen Cholinesterasehemmer und Memantine im Vordergrund. Bei der Behandlung von Verhaltensauffälligkeiten besteht unter Neuroleptika ein erhöhtes Schlaganfallrisiko, das bei der Behandlung bedacht werden sollte.

ag der Anteil der über 60-Jährigen 1995 in Deutschland noch bei $21 \%$, wird er aufgrund der steigenden Lebenserwartung bis 2040 vermutlich auf etwa 36\% ansteigen. Damit wird auch die Zahl der Demenzkranken weiter zunehmen. Bereits heute leben mehr als eine Million an Demenz erkrankte Menschen in Deutschland. Bis 2040 wird sogar eine Verdoppelung bis Verdreifachung der Anzahl erwartet. Als häufigste Demenzform gilt die Alzheimer-Demenz (etwa 60\%), gefolgt von der vaskulären Demenz (VaD) mit etwa 15 bis 20\% und der Demenz mit Lewy-Körperchen (10\%).

Bei genauer Betrachtung ist allerdings festzustellen, dass fast alle dementen Patienten über 60 Jahre sowohl neurodegenerative als auch vaskuläre Hirnveränderungen aufweisen. Auch bei Alzheimer-Demenz können vaskuläre Läsionen vorliegen. Alzheimer-Patienten mit Läsionen schneiden dabei in kognitiven Tests deutlich schlechter ab und weisen eine schlechtere Prognose auf als Patienten ohne Läsionen (13).

\section{Ursachen}

Als Risikofaktoren, die auch das Risiko für vaskuläre Erkrankungen erhöhen, konnten u.a. bereits identifiziert werden (nach 2):

- Alter

- Thrombogene Faktoren

- Atherosklerose

- ApoE4

- Schlaganfall

- Hohes Serum-Homocystein

- Diabetes mellitus

- Hypertension

- Rauchen

- Hypotension

- Alkoholismus

- Hoher Fibrinogenspiegel

- Hohes LDL-Cholesterin

- Kopfverletzungen/Bewusstseinsstörungen

- Herzerkrankungen

- Menopause

- Migräne

- Niedriger Bildungsstand

- Hohe Serumviskosität

- Transiente ischämische Attacken

- Depression

- Pathologische Veränderungen der Mikrogefäße

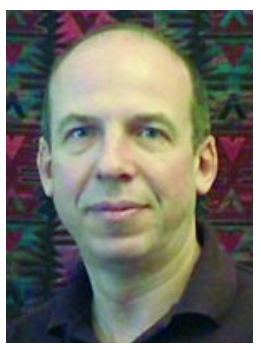

Georg Adler
- $\quad$ Zu fettreiche Ernährung

- Erhöhung des C-reaktiven Proteins.

Im Mittelpunkt der Erkrankung scheinen die Amyloid-Ablagerungen und artherosklerotische Veränderungen $\mathrm{zu}$ stehen, die bereits von Alois Alzheimer in seiner Beschreibung einer „eigenartigen Erkrankung der Hirnrinde“ erwähnt wurden. Beta- und Gammasekretasen spalten das Amyloid-Präkursorprotein (APP) in kleinere Fragmente, z.B. das zelltoxische Abeta1-42, die zum Teil abgelagert werden oder über den Liquorraum eliminiert werden. Während bei Gesunden gebildetes Amyloid-beta teilweise von Makrophagen beseitigt wird, ist dieser Prozess bei Demenzpatienten beeinträchtigt (6). Amyloid-beta verringert außerdem die glutamaterge Neurotransmission und synaptische Plastizität. Seit kurzem wird deutlich, dass die zellulären Mikrogefäße dabei eine entscheidende Rolle spielen. Die zerebrale Hypoperfusion, die bei Alzheimer-Patienten beobachtet wird (18), und u.a. einen Sauerstoff- und Glukosemangel bewirkt, führt $\mathrm{zu}$ Schäden in den Mikrogefäßen (5), die dann offensichtlich zelltoxische Verbindungen freisetzen (11).

Diagnostik

Störungen des Gedächtnisses, Denkvermögens und der emotiona- 
Tab. 1 Obligate und fakultative Lapordiagnostik sowie apparative Untersuchungen bei Demenzverdacht

(nach 6)

obligat (diagnostisches

Minimalprogramm)

Laboruntersuchungen

Blutbild, Differenzialblutbild

Glukose

Cholesterin

$\mathrm{Na}, \mathrm{K}, \mathrm{Ca}$

Gamma-GT

Kreatinin

TSH

Vitamin B12, Folsäure

Blutsenkung

\section{Apparative Verfahren}

EKG

MR oder CT fakultativ (erweiterte Diagnostik bei entsprechendem Verdacht)

Urinstatus, Lues-, HIV-, Borrelienserologie

HbA1C, Blutzuckertagesprofil

Lipidelektropherese

$\mathrm{Cl}, \mathrm{Mg}, \mathrm{Cu}$

GOT, GPT, alkalische Phosphatase, Bilirubin, Coeruloplasmin

Harnstoff, Harnsäure

T3, T4, Antikörper, Parathormon

Homocystein, Thiamin

C-reaktives Protein, ANA; ANCA, AMA, Liquordiagnostik, HIVTest, Untersuchung auf toxische Substanzen (Blei, Kupfer, Quecksilber, Benzol, Toluol, u. a.), Drogenscreening (z.B. Benzodiazepine)

Langzeit EKG, 24-h-RR, Echokardiografie, Dopplersonografie, EEG, evozierte Otentiale, Polysomnografie SPECT, PET len Kontrolle müssen nach ICD-10 mehr als sechs Monate anhalten und so ausgeprägt sein, dass die Aktivitäten des täglichen Lebens erheblich beeinträchtigt sind. Falls möglich, sollte der Patient selbst zu seiner Anamnese Auskunft geben, bei deutlicheren Störungen des Gedächtnisses ist die Befragung der Angehörigen unverzichtbar. Neben der internistischen, neurologischen und laborchemischen Untersuchung (Tab. 1) sollten stets neuropsychologische Tests durchgeführt werden. Mittlerweile haben sich z.B. der DemTect ${ }^{\circledR}$ Demenz-Detections-Test (10 min, auch im frühen Stadium geeignet), MiniMental-Status-Test (MMST, $10 \mathrm{~min}$, bei leichter Störung wenig geeignet), Syndrom-Kurz-Test (SKT, $10 \mathrm{~min}$, auch im frühen Stadium geeignet) und das Structured Interview for the Diagnosis of dementia of the Alzheimer type, Multiinfarct dementia and dementias of other aetiology (SIDAM) etabliert. Zum Ausschluss von Gehirntumoren, Infarkten und anderen fokalen Hirnveränderungen sollten eine Computertomografie (CT) oder Magnetresonanztomografie (MRT) aufgenommen werden.

\section{Therapie}

Grundlage der Therapie ist ein multimodales Behandlungskonzept. Insbesondere der Hausarzt ist gefordert. Körperliche Krankheiten, wie Diabetes mellitus oder Bluthochdruck, können demenzielle Symptome verstärken und sogar hervor- rufen. Neben der Behandlung von Krankheitsursachen ist eine pharmakologische Therapie der demenziellen und eventuell auch von begleitenden Verhaltensaufälligkeiten erforderlich. Auch die nichtmedikamentöse Therapie, z.B. Milieutherapie, Psychoedukation, verbessert die Lebensqualität der Betroffenen und ihrer Angehörigen, allerdings besteht noch wenig wissenschaftliche Evidenz.

Zur medikamentösen Behandlung werden heute Cholinesterasehemmer (Galantamin, Rivastigmin, Donepezil) und der NMDA-Rezeptorantagonist Memantine eingesetzt. Die Wirksamkeit der Cholinesterasehemmer wurde im letzten Jahr kontrovers diskutiert, dies ist auf methodische Eigenarten der AD2000-Studie mit Donepezil (1) zurückzuführen, in der zuletzt nur noch vier Patienten eingeschlossen waren! Die Ergebnisse stehen in Konflikt mit allen bisher durchgeführten Studien mit Donepezil (7). Hinzu kommt, dass der individuelle Krankheitsverlauf bei Demenz unglaublich variabel ist, sodass aus dem individuellen Verlauf keine Rückschlüsse auf die Wirksamkeit des eingesetzten Medikaments möglich sind.

Zur Behandlung der Verhaltensänderungen (Tab. 2), die als häufigster Grund für eine Heimeinweisung angegeben werden, werden zunehmend atypische Neuroleptika (Risperidon, Olanzapin, Quetiapin, Amisulprid, Aripiprazol, Ziprasidon) eingesetzt, wobei allerdings nur Risperidon (Risperdal ${ }^{\circledR} 1 \mathrm{mg}$ ) für die Therapie von Verhaltensauffälligkeiten bei Demenzerkrankungen zugelassen

Tab. 2 Verhaltensauffälligkeiten bei Demenz

\begin{tabular}{|c|c|c|c|c|}
\hline Symptom & Alzheimer Krankheit & Vaskuläre Demenz & $\begin{array}{l}\text { Diffuse Lewy- } \\
\text { Body-Demenz }\end{array}$ & $\begin{array}{c}\text { Frontotemporale } \\
\text { Demenz }\end{array}$ \\
\hline Psychomotorische Agitiertheit & +++ & +++ & +++ & + \\
\hline Aggressives Verhalten & ++ & ++ & ++ & + \\
\hline Wahnvorstellungen & ++ & ++ & +++ & + \\
\hline Halluzinationen & + & + & +++ & - \\
\hline Depression & ++ & +++ & ++ & + \\
\hline Angst & ++ & +++ & + & + \\
\hline Apathie/Verlangsamung & ++ & +++ & ++ & ++++ \\
\hline Schlafrhythmusveränderungen & ++ & ++ & ++ & +++ \\
\hline Appetitveränderungen & + & + & + & +++ \\
\hline Sexuelle Enthemmung & + & + & + & +++ \\
\hline
\end{tabular}


ist. Die Wirksamkeit wird allgemein als gut eingeschätzt, wobei die einzelnen atypischen Neuroleptika offenbar ähnlich abschneiden, wie in der „Clinical Antipsychotic Trials in Intervention Effectiveness-Alzheimer's Disease Study (CATIE-AD)“Studie beobachtet wurde, die vom National Insitute for Mental Health gefördert wurde (20).

Atypische Neuroleptika können jedoch bei Demenzpatienten das Risiko für kardiovaskuläre Ereignisse erhöhen (siehe auch Beitrag von Fritze in diesem Heft), in den USA hat daher die amerikanische Zulassungsbehörde FDA im Mai 2005 vorerst nur für die atypischen Neuroleptika entsprechende Warnhinweise („boxed warning“) verfügt. Eine retrospektive Analyse der Daten von fast 33000 Patienten hat jetzt auch ergeben, dass das Schlaganfallrisiko unter atypischen Neuroleptika vergleichbar ist (etwa 1,5\%) mit dem von konventionellen Neuroleptika wie Haloperidol, Fluphenazin oder Chlorpromazin (10). Neuroleptika, aber auch Sedativa, können die bei Demenz-Erkrankungen vorliegende zerebrale Hypoperfusion weiter verschlechtern und so $\mathrm{zu}$ Mikroläsionen und toxischen Prozessen führen, die die Demenz weiter voran treiben (15). Der Einsatz von Neuroleptika bei Demenzpatienten sollte daher vorsichtig abgewogen werden. Besserungen der Verhaltensauffälligkeiten wurden auch unter den Cholinesterasehemmern und Memantine nachgewiesen (z.B. 8)

\section{- Neue Therapieansätze}

Neue Therapieansätze bei der $\mathrm{AD}$ beruhen vorwiegend auf der Amyloid-Hypothese. So konnte bereits gezeigt werden, dass Statine (3) eine Reihe von pleiotropen, gefäßprotektiven Wirkungen aufweisen, wie Schutz vor oxidativem Stress, erhöhte Bioverfügbarkeit von Stickoxid (NO), Schutz vor Entzündungen und auch die Verbesserung des zerebralen Blutflusses. Statine scheinen dabei auch die Bildung von Amyloidablagerungen zu verhindern, wobei der genaue Mechanismus noch unklar ist. Auch die Einnahme von nicht-steroidalen Antirheumatika,
Vitamin E, Deprenyl und einige Antirheumatika verringern offenbar das Risiko einer Alzheimer-Erkrankung, vermutlich über entzündungshemmende Mechanismen, die an der Amyloidbildung beteiligt sind (14). Beispielsweise wird Ibuprofen zurzeit in Phase-III-Studien untersucht. Allerdings hat bisher noch keine prospektive, randomisierte Studie eine Wirksamkeit von nichtsteroidalen Antirheumatika (NSARs) bei Demenz nachweisen können. Retrospektiv scheinen aber NSARs Demenzerkrankungen hinauszuzögern (19).

Auf die Amyloidablagerungen konzentrieren sich auch die Forschungen mit Beta- und Gamma-Sekretase-Inhibitoren. Eine Hemmung der Sekretasen führt zu einer verminderten Bildung des toxischen Abeta1-42-Fragments. Bisher sind jedoch die entsprechenden Kandidaten noch im präklinischen Stadium. Weiter ist bereits NC-531, ein Glycosaminoglycan, das die Bildung von Amyloidablagerungen und die Bildung von Fibrillen verhindern soll und zurzeit in Phase-III-Studien untersucht wird (9).

Im Tiermodell ist auch eine aktive Immunisierung gegen das $\mathrm{Ab}$ bauprodukt des Amyloid-Präkursorproteins, Abeta1-42, möglich. Klinische Studien mussten allerdings aufgrund von Menigoenzephalitiden abgebrochen werden. Zurzeit wird an einer Impfung mit anderen Derivaten des Amyloid-Präkursorproteins gearbeitet (15).

Vielversprechend sind auch neurotrophe Faktoren. NeoTrofin (Phase II/III) und Cerebrolysin (Phase II/III) sollen die Wirkung des Nerve Growth Factor (NGF) nachahmen. In Studien konnte bereits eine signifikante Verbesserung von Gedächtnis und Konzentration unter Cerebrolysin bestätigt werden (z.B. 17).

\section{Treatment of Dementia - An Update}

Vascular factors are causally involved in Alzheimer's disease. The cerebral capillaries apparently play a major role in the evolution of the disease. For this reason, diagnostic measures should also check for the presence of hypertension, thyroid gland parameters and diabetes mellitus, as well as other risk factors. With regard to treatment, a multimodal approach is recommended. For medicinal therapy, cholinesterase inhibitors and memantine are of primary importance. The use of neuroleptic agents is associated with an increased risk of stroke, and this must be taken into account when treatment is applied.

\section{Key words}

dementia - treatment - diagnosis amyloid-beta

\section{Literatur}

1. Courtney C, Farrell D, Gray R, Hills R, Lynch L, Sellwood E, Edwards S, Hardyman W, Raftery J, Crome P, Lendon C, Shaw H, Bentham P; AD2000 Collaborative Group. Long-term donepezil treatment in 565 patients with Alzheimer's disease (AD2000): randomised double-blind trial. Lancet 2004; 363 (9427): 2105-15

2. de la Torre JC. Alzheimer disease as a vascular disorder: nosological evidence. Stroke 2002; 33 (4): 1152-62

3. Endres M. Statins and stroke. I Cereb Blood Flow Metab 2005; 25 (9): 1093-110

4. Farkas E, De Vos RA, Jansen Steur EN, Luiten PG. Are Alzheimer's disease, hypertension, and cerebrocapillary damage related? Neurobiol Aging 2000; 21: 235-243

5. Fiala M. Ineffective phagocytosis of amyloid-beta by macrophages of Alzheimer's disease patients. J Alzheimers Dis 2005; 7 (3): 221-32

6. Förstl H, Einhäuptl KM. Diagnose und Differentialdiagnose der Demenzen. In Beyreuther K, Einhäuptl KM, Förstl H, Kurz A (Hrsg.). Demenzen. Grundlagen und Klinik. Stuttgart, Thieme-Verleg, 2002: 43-70

7. Fritze J, Gastpar M. Demenzkranke bitte nicht diskriminieren. Stellungnahme zur Kritik am Wirksamkeitsnachweis von Cholinesterasehemmern. psychoneuro 2004; 30 (9): 512-513

8. Gauthier S, Wirth Y, Mobius HJ. Effects of memantine on behavioural symptoms in Alzheimer's disease patients: an analysis of the Neuropsychiatric Inventory (NPI) data of two randomised, controlled studies Int J Geriatr Psychiatry 2005; 20: 459

9. Geerts H. NC-531 (Neurochem). Curr Opin Investig Drugs 2004; 5 (1): 95-100

10. Gill SS et al. Atypical antipsychotic drugs and risk of ischaemic stroke: population based retrospective cohort study BM] 2005; 330: 445

11. Grammas P et al. Microvessels from Alzheimer's disease brains kill neurons in vitro. Am J Pathol 1999; 154 (2): 337-42

12. Kalaria RN. Small vessel disease and Alzheimer's dementia: pathological considerations. Cerebrovasc Dis 2002; 13: S48-52

13. Kalaria RN. The role of cerebral ischemia in Alzheimer's disease. Neurobiol Aging 2000; 21: 321-330 


\section{Schwerpunkt / Bücher}

14. Klegeris und McGeer. Non-steroidal anti-inflammatory drugs (NSAIDs) and other anti-inflammatory agents in the treatment of neurodegenerative disease. Curr Alzheimer Res 2005; 2 (3): 355-65

15. Klyubin et al. Amyloid beta protein immunotherapy neutralizes Abeta oligomers that disrupt synaptic plasticity in vivo. Nat Med 2005; 11 (5): 556-561

16. Miklossy J. Cerebral hypoperfusion induces cortical watershed microinfarcts which may further aggravate cognitive decline in Alzheimer's disease. Neurol Res 2003; 25 (6): 605-10
17. Rockenstein E, Adame A, Mante M, Moessler $\mathrm{H}$, Windisch $\mathrm{M}$, Masliah $\mathrm{E}$. The neuroprotective effects of Cerebrolysin trade mark in a transgenic model of Alzheimer's disease are associated with improved behavioral performance. J Neural Transm 2003; 110 (11):1313-27

18. Ruitenberg A, den Heijer T, Bakker SL, van Swieten JC, Koudstaal PJ, Hofman A, Breteler MM. Cerebral hypoperfusion and clinical onset of dementia: the Rotterdam Study. Ann Neurol 2005; 57 (6): 789-94 19. Tabet N, Feldman H. Ibuprofen for Alzheimer's disease (Cochrane Review). Coch- rane Database Syst Rev 2003. CD004031 20. The NIMH Clinical Antipsychotic Trial of Intervention Effectiveness for Alzheimer's Disease (CATIE-AD): First Outcomes. APA 2005

\section{Korrespondenzadresse: \\ Prof. Dr. med. Georg Adler}

Zentralinstitut für Seelische Gesundheit 15

68159 Mannheim

adler@zi-mannheim.de 